\title{
一类运算图的匹配数 ${ }^{*}$
}

\author{
晏卫根 ${ }^{1,2}$ 叶永南 ${ }^{2}$
}

(1. 集美大学理学院, 厦门 $361021 ; 2$. 台湾中央研究院数学研究所, 台北 11529)

摘要 设 $G$ 是一个简单图, 把 $G$ 的每条边 $e=(a, b)$ 变换成一个三角形 $a e^{*} b$ 而 得到一个新图, 记为 $R(G)$, 其中新增加的顶点 $e^{*}$ 的度为 2. 本文证明 $R(G)$ 的匹 配数完全由图 $G$ 的顶点度序列确定.

\section{关键词 匹配 Hosoya 指标 匹配多项式 定向关联矩阵 反邻接矩阵}

\section{1 引言}

除特别说明外，本文假设 $G=(V(G), E(G))$ 是一个简单图，其顶点集为 $V(G)=\left\{v_{1}, v_{2}, \ldots, v_{n}\right\}$, 边集为 $E(G)=\left\{e_{1}, e_{2}, \ldots, e_{m}\right\}$. 对 $G$ 的任意一个顶点 $v_{i}$, 用 $d_{G}\left(v_{i}\right)$ 或 $d_{i}$ 表示 $G$ 中顶点 $v_{i}$ 的度, 即 $G$ 中与 $v_{i}$ 相关联的边的数目, 而 $D(G)=\operatorname{diag}\left(d_{1}, d_{2}, \ldots, d_{n}\right)$ 表示 $G$ 的顶点度对角矩阵. 设 $A(G)=\left(a_{i j}\right)_{n \times n}$ 是一 个 $n$ 阶矩阵, 其中如果顶点 $v_{i}$ 与 $v_{j}$ 相邻, 则 $a_{i j}$ 等于 1 , 否则 $a_{i j}$ 等于 0 , 称 $A(G)$ 为 图 $G$ 的邻接矩阵. 如果 $G^{e}$ 是 $G$ 的一个定向, 定义一个 $n$ 阶矩阵 $A\left(G^{e}\right)=\left(b_{i j}\right)_{n \times n}$ 如下:

$$
b_{i j}= \begin{cases}1, & \text { 当 }\left(v_{i}, v_{j}\right) \in E\left(G^{e}\right) \text { 时, } \\ -1, & \text { 当 }\left(v_{j}, v_{i}\right) \in E\left(G^{e}\right) \text { 且 } i \neq j \text { 时, } \\ 0, & \text { 其他. }\end{cases}
$$

称 $A\left(G^{e}\right)$ 为图 $G$ 的定向 $G^{e}$ 的反邻接矩阵 ${ }^{[1]}$, 显然 $A\left(G^{e}\right)$ 是一个 $n$ 阶反对称 矩阵, 即 $A\left(G^{e}\right)^{\mathrm{T}}=-A\left(G^{e}\right)$. 图 $G$ 的定向 $G^{e}$ 的定向关联矩阵 (oriented incident

*福建省青年科技人才创新项目 (批准号: 2004J024), 福建省自然科学基金 (批准号:E0540007), 台湾 自然科学基金 (批准号:94-M001-017) 资助项目

**E-mail: weigenyan@263.net(W. Yan); mayeh@math.sinica.edu.tw(Y.-N. Yeh) 
matrix) 记为 $M\left(G^{e}\right)=\left(c_{i j}\right)_{n \times m}$, 其中

$$
c_{i j}= \begin{cases}1, & \text { 如果 } e_{j} \text { 是顶点 } v_{i} \text { 的出孤, } \\ -1, & \text { 如果 } e_{j} \text { 是顶点 } v_{i} \text { 的入孤, } \\ 0, & \text { 其他. }\end{cases}
$$

由文献 [2] 知, $M\left(G^{e}\right) M\left(G^{e}\right)^{\mathrm{T}}=D(G)-A(G)$, 此矩阵称为图 $G$ 的 Laplace 矩阵.

设 $e=(u, v)$ 是 $G$ 的一条边 (方便起见, 有时也记为 $e=u v$ ), $G-u-v$ 表示 $G$ 中删去顶点 $u$ 与 $v$ 得到的顶点导出子图, $G-e$ 表示 $G$ 中删去边 $e$ 得到的 $G$ 的边导出子图. 图 $G$ 的一个边子集 $M$ 称为一个匹配 (matching), 如果 $G$ 中的每个 顶点与 $M$ 中的至多一条边相关联; $M$ 称为 $G$ 的一个完美匹配 (perfect matching), 如果 $G$ 中的每个顶点恰好与 $M$ 中的一条边相关联. 记 $m(G, j)$ 为 $G$ 中含有 $j$ 条 边的匹配的数目, 习惯上总假定 $G$ 的零匹配数为 1 , 即 $m(G, 0)=1$, 于是 $m(G, 1)$ 为图 $G$ 的边数; 而当 $n$ 是偶数时, $m\left(G\right.$, $\left.\left[\frac{n}{2}\right]\right)$ 则为 $G$ 的完美匹配的数目. 用 $Z(G)$ 表示图 $G$ 中所有匹配的数目, 即

$$
Z(G)=m(G, 0)+m(G, 1)+\cdots+m\left(G,\left[\frac{n}{2}\right]\right),
$$

图 $G$ 中所有匹配的数目也称为图 $G$ 的 Hosoya 指标 (见文献 [3]), 多项式

$$
m(G, x)=\sum_{j=0}^{\left[\frac{n}{2}\right]}(-1)^{j} m(G, j) x^{n-2 j}
$$

称为图 $G$ 的匹配多项式 (matching polynomial). 由 $Z(G)$ 与 $m(G, x)$ 的定义, 显然 $Z(G)=\left.\frac{1}{i^{n}} m(G, x)\right|_{x=i}$, 其中 $i^{2}=-1$. 因为在物理与化学中有许多应用, 图的匹 配数 (Hosoya 指标) 以及匹配多项式不但为数学家, 而且也被物理学家与化学家 进行过广泛与深入的研究 (见文献 [4-15]). 匹配是图论中的一个经典概念, 它反 复在组合学中出现, 图的匹配数作为一个拓扑指标在化学中的应用, 首次由化 学家 Hosoya ${ }^{[3]}$ 提出, 他以此描述饱和碳氢化合物的热力学性质. 图的匹配数以 及匹配多项式的计算是困难的—NP 完全问题 (见文献 [16]), 与此相关的工作 见文献 [17-21].

设 $G=(V(G), E(G))$ 是一个简单图, 定义一个新图 $R(G)$ (见文献 [2] 中第 63 页的定义), 使得 $R(G)=(V(R(G)), E(R(G)))$ 是由图 $G$ 实施如下运算得到的图: 对 $G$ 的每条边 $e=(a, b)$, 增加一个新顶点 $e^{*}$, 并连边 $a e^{*}$ 与 $b e^{*}$. 换句话说, 把 $G$ 的 每条边 $e=(a, b)$ 变换成一个三角形 $a e^{*} b$ 而得到的图就是 $R(G)$, 其中新增加的顶 点 $e^{*}$ 是一个二度顶点. 因此 $R(G)$ 的顶点集为 $V(R(G))=V(G) \cup\left\{e^{*} \mid e \in E(G)\right\}$, 边集为 $E(R(G))=E(G) \cup\left\{\left(v_{r}, e^{*}\right),\left(v_{s}, e^{*}\right) \mid e=\left(v_{r}, v_{s}\right) \in E(G)\right\}$. 图 1(a) 与 (b) 说 明了由图 $G$ 构造图 $R(G)$ 的方法.

本文的主要结果是:

定理 1 设 $G$ 是有 $n$ 个顶点的简单图, $R(G)$ 是上面定义的图, 则 $R(G)$ 的 
匹配数可以表示为

$$
Z(R(G))=\left(d_{1}+1\right)\left(d_{2}+1\right) \cdots\left(d_{n}+1\right),
$$

其中 $\left(d_{1}, d_{2}, \ldots, d_{n}\right)$ 是图 $G$ 的顶点度序列.

我们将给出两种证明上面定理的方法：一种是归纳法; 另一种是一一对应 法.

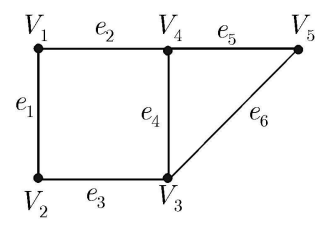

(a)

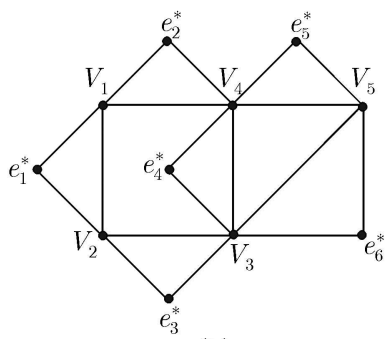

(b)

图 1 (a) 图 $G$, (b) 对应于图 $G$ 的图 $R(G)$

\section{2 定理 1 的证明}

为了证明定理 1 , 需要引入一些引理.

引理 $\mathbf{2}^{[22]}$ 设 $G$ 是一个图, $e=(a, b)$ 是 $G$ 的一条边, 则

$$
Z(G)=Z(G-a b)+Z(G-a-b),
$$

其中 $G-a b$ 与 $G-a-b$ 分别表示 $G$ 中删去边 $e$ 及删去顶点 $u$ 与 $v$ 的边导出子图 与顶点导出子图.

设 $G=(V(G), E(G))$ 是有 $n$ 个顶点的图， $a$ 是 $\mathrm{G}$ 的一个顶点. 构造一个 $n+2$ 个顶点的新图 $G^{\prime}=\left(V\left(G^{\prime}\right), E\left(G^{\prime}\right)\right)$, 使得 $G^{\prime}$ 的顶点集为 $V\left(G^{\prime}\right)=V(G) \cup\{x, y\}$, 边集为 $E\left(G^{\prime}\right)=E(G) \cup\{(a, x),(a, y),(x, y)\}$, 其中 $x, y \notin V(G)$ (见图 2(a) 与 (b)).

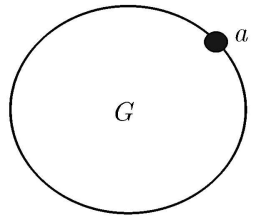

(a)

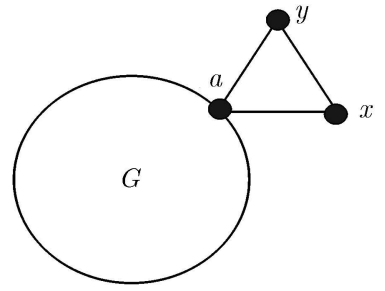

(b)

图 2 (a) 图 $G$, (b) 对应于图 $G$ 的图 $G^{\prime}$

引理 3 设 $G$ 是有 $n$ 个顶点的简单图, $G^{\prime}$ 是上面定义的图, 则

$$
Z\left(G^{\prime}\right)=2 Z\left(G^{\prime}-x\right)=2 Z\left(G^{\prime}-y\right) .
$$


证明 由引理 2,

$$
\begin{aligned}
& Z\left(G^{\prime}\right)=Z\left(G^{\prime}-x-y\right)+Z\left(G^{\prime}-x y\right)=Z(G)+Z\left(G^{\prime}-x y\right), \\
& Z\left(G^{\prime}-x y\right)=Z(G-a)+Z\left(G^{\prime}-x\right), Z\left(G^{\prime}-x\right)=Z(G-a)+Z(G),
\end{aligned}
$$

因此,

$$
Z\left(G^{\prime}\right)=Z(G)+Z(G-a)+Z\left(G^{\prime}-x\right)=2 Z\left(G^{\prime}-x\right) .
$$

可以类似地证明 $Z\left(G^{\prime}\right)=2 Z\left(G^{\prime}-y\right)$, 证毕.

引理 $4^{[5,12]}$ 设 $G$ 是一个没有偶长圈的 $n$ 个顶点的简单图, $G^{e}$ 是 $G$ 的任 意一个定向, 则 $G$ 的匹配数为

$$
Z(G)=\operatorname{det}\left(I_{n}+A\left(G^{e}\right)\right)
$$

其中 $I_{n}$ 是 $n$ 阶单位矩阵, $A\left(G^{e}\right)$ 是图 $G$ 的定向 $G^{e}$ 的反邻接矩阵.

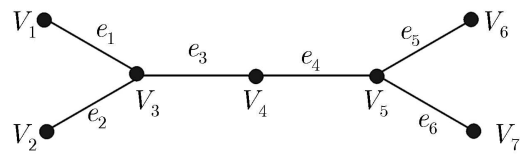

(a)

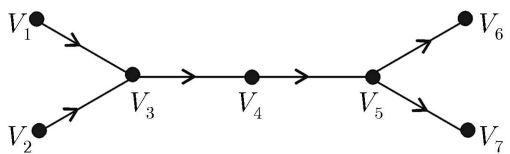

(b)

图 3 (a) 证明引理 5 中的树 $T$, (b) 树 $T$ 的定向 $T^{e}$

引理 5 设 $T$ 是一棵 $n$ 个顶点的树, 则 $R(T)$ 的匹配数可表示为

$$
Z(R(T))=\left(d_{1}+1\right)\left(d_{2}+1\right) \cdots\left(d_{n}+1\right),
$$

其中 $\left(d_{1}, d_{2}, \ldots, d_{n}\right)$ 是树 $T$ 的顶点度序列.

证明 首先给 $T$ 一个定向 $T^{e}$ 如下: 对 $T$ 的每一条边 $e=\left(v_{i}, v_{j}\right)$, 如果 $i<j$, 则定义 $e$ 的方向是从 $v_{i}$ 指向 $v_{j}$, 否则 $e$ 的方向是从 $v_{j}$ 指向 $v_{i}$. 对于图 3(a) 中的树 $T$, 其相应的定向 $T^{e}$ 见图 3(b).

利用 $T^{e}$ 来定义 $R(T)$ 的一个定向 $R(T)^{e}$ 如下: 注意到 $R(T)$ 有两种类型的 边, 一种为 $\left(v_{i}, v_{j}\right)$ 形式的边 (见图 4(a)), 这类边可以看作 $T$ 中的边; 另一种为形 式 $\left(v_{k}, e_{l}^{*}\right)$ 的边 (其中假设边 $e_{l}$ 的两个端点是 $v_{k}$ 与 $v_{s}$ ). 定义第 1 类边 $\left(v_{i}, v_{j}\right)$ 的方 向与其在 $T^{e}$ 中的方向相同, 定义第 2 种形式的边 $\left(v_{k}, e_{l}^{*}\right)$ 的方向为: 如果 $k<s$, 则 $v_{k}$ 指向 $e_{l}^{*}$, 如果 $k>s$, 则 $e_{l}^{*}$ 指向 $v_{k}$. 经过这样的步骤得到 $R(T)$ 的一个定向就 是 $R(T)^{e}$. 对于图 3(a) 中的树 $T$, 其对应的 $R(T)$ 的定向 $R(T)^{e}$ 见图 4(b).

经过对 $R(T)^{e}$ 的顶点进行恰当的标号, 其反邻接矩阵 $A\left(R(T)^{e}\right)$ 具有如下形 式:

$$
A\left(R(T)^{e}\right)=\left(\begin{array}{cc}
A\left(T^{e}\right) & M\left(T^{e}\right) \\
-M\left(T^{e}\right)^{T} & 0
\end{array}\right),
$$

其中 $A\left(T^{e}\right)$ 与 $M\left(T^{e}\right)$ 分别表示 $T^{e}$ 的反邻接矩阵 (skew adjacency matrix) 与定向关 联矩阵 (oriented incident matrix). 


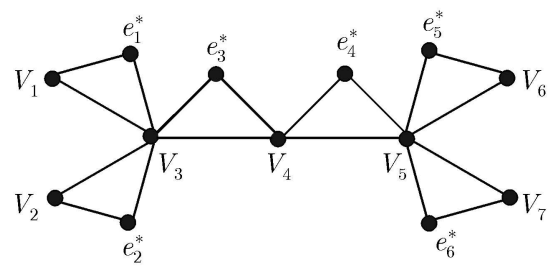

(a)

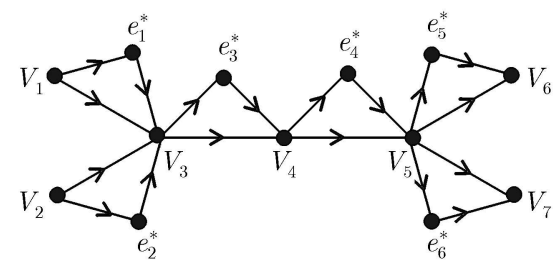

(b)

图 4 (a) 图 3(a) 中的树 $T$ 对应的图 $R(T)$, (b) $R(T)$ 的定向 $R(T)^{e}$

因为 $T$ 是一棵树, 由 $R(T)$ 的定义, 它不包含长度为偶数的圈. 由引理 4 得,

$$
\begin{aligned}
Z(R(T)) & =\operatorname{det}\left(I_{2 n-1}+A\left(R(T)^{e}\right)\right)=\operatorname{det}\left(\begin{array}{cc}
I_{n}+A\left(T^{e}\right) & M\left(T^{e}\right) \\
-M\left(T^{e}\right)^{T} & I_{n-1}
\end{array}\right) \\
& =\operatorname{det}\left(I_{n}+A\left(T^{e}\right)+M\left(T^{e}\right) M\left(T^{e}\right)^{T}\right) \\
& =\operatorname{det}\left(I_{n}+A\left(T^{e}\right)+D(T)-A(T)\right),
\end{aligned}
$$

其中 $D(T)$ 与 $A(T)$ 分别是 $T$ 的顶点度对角矩阵与邻接矩阵. 由 $T^{e}$ 的定义, $A\left(T^{e}\right)$ 中的对角线上面的元素不小于零, 而对角线下面的元素不大于零, 因此 $A\left(T^{e}\right)-A(T)$ 是一个下三角矩阵, 所以

$$
Z(R(T))=\operatorname{det}\left(I_{n}+D(T)+A\left(T^{e}\right)-A(T)\right)=\left(d_{1}+1\right)\left(d_{2}+1\right) \cdots\left(d_{n}+1\right) .
$$

至此, 完成了引理的证明.

由引理 5,下面的推论是显然的:

推论 6 设 $G$ 是 $n$ 个顶点的无圈图, 则它的匹配数可表示为

$$
Z(R(G))=\left(d_{1}+1\right)\left(d_{2}+1\right) \cdots\left(d_{n}+1\right),
$$

其中 $\left(d_{1}, d_{2}, \ldots, d_{n}\right)$ 是 $G$ 的顶点度序列.

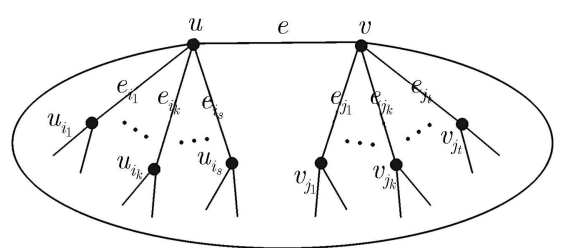

(a)

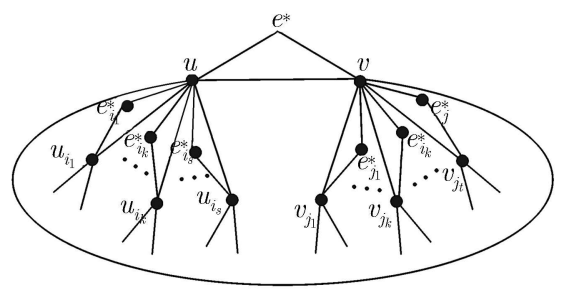

(b)

图 5 (a) 图 $G$, (b) 图 $G$ 对应的图 $R(G)$

定理 1 的归纳法证明 对 $G$ 的基圈数 (cyclomatic number) 进行归纳法证明. 如果 $G$ 是一个无圈图, 由上面的推论 6 , 定理成立. 下面假设 $G$ 包含圈. 设 $e=(u, v)$ 是 $G$ 的一条边, 它在 $G$ 的某个圈上, $\left\{u_{i_{1}}, u_{i_{2}}, \ldots, u_{i_{s}}, v\right\}$ 与 $\left\{v_{j_{1}}, v_{j_{2}}, \ldots, v_{j_{t}}, u\right\}$ 
分别是 $u$ 与 $v$ 的邻点集 (见图 5(a)). 为方便起见, 令 $e_{i_{k}}=\left(u, u_{i_{k}}\right)(1 \leqslant k \leqslant s$ ), $e_{j_{k}}=\left(v, v_{j_{k}}\right)(1 \leqslant k \leqslant t)$, 因此 $d_{G}(u)=s+1, d_{G}(v)=t+1$. 于是只需要证明

$$
Z(R(G))=(s+2)(t+2) \prod_{w \in V(G), w \neq u, v}\left(d_{G}(w)+1\right) .
$$

先定义 3 个相关图 $G_{u}, G_{v}$ 与 $G_{u v}$ 如下:

(i) $G_{u}$ 是有 $n-1+s$ 个顶点的图, 它是从 $G$ 中删去顶点 $u$ 与增加 $s$ 条悬挂 边 $\left(u_{i_{1}}, e_{m_{1}}^{\prime}\right),\left(u_{i_{2}}, e_{m_{2}}^{\prime}\right), \ldots,\left(u_{i_{s}}, e_{m_{s}}^{\prime}\right)$ 而得到的图;

(ii) $G_{v}$ 是有 $n-1+t$ 个顶点的图, 它是从 $G$ 中删去顶点 $v$ 与增加 $t$ 条悬挂 边 $\left(v_{j_{1}}, e_{n_{1}}^{\prime}\right),\left(v_{j_{2}}, e_{n_{2}}^{\prime}\right), \ldots,\left(v_{j_{t}}, e_{n_{t}}^{\prime}\right)$ 而得到的图;

(iii) $G_{u v}$ 是有 $n-2+s+t$ 个顶点的图, 它是从 $G$ 中删去顶点 $u$ 与 $v$, 同时增加 $s+t$ 条悬挂边 $\left(u_{i_{1}}, e_{m_{1}}^{\prime}\right),\left(u_{i_{2}}, e_{m_{2}}^{\prime}\right), \ldots,\left(u_{i_{s}}, e_{m_{s}}^{\prime}\right),\left(v_{j_{1}}, e_{n_{1}}^{\prime}\right),\left(v_{j_{2}}, e_{n_{2}}^{\prime}\right), \ldots,\left(v_{j_{t}}, e_{n_{t}}^{\prime}\right)$ 而得到的图.

因为 $e$ 是 $G$ 中某个圈上的一条边, 因此 4 个图 $G-e, G_{u}, G_{v}$ 与 $G_{u v}$ 的基圈 数比 $G$ 的基圈数少. 由归纳法得

$$
\begin{gathered}
Z(R(G-e))=\prod_{w \in V(R(G-e))}\left(d_{R(G-e)}(w)+1\right) \\
=(s+1)(t+1) \prod_{w \in V(G), w \neq u, v}\left(d_{G}(w)+1\right) ; \\
Z\left(R\left(G_{u}\right)\right)=\prod_{w \in V\left(G_{u}\right)}\left(d_{G_{u}}(w)+1\right)=2^{s}(t+1) \prod_{w \in V(G), w \neq u, v}\left(d_{G}(w)+1\right) ; \\
Z\left(R\left(G_{v}\right)\right)=\prod_{w \in V\left(G_{v}\right)}\left(d_{G_{v}}(w)+1\right)=2^{t}(s+1) \prod_{w \in V(G), w \neq u, v}\left(d_{G}(w)+1\right) ; \\
Z\left(R\left(G_{u v}\right)\right)=\prod_{w \in V\left(G_{u v}\right)}\left(d_{G_{u v}}(w)+1\right)=2^{s+t} \prod_{w \in V(G), w \neq u, v}\left(d_{G}(w)+1\right) .
\end{gathered}
$$

另一方面, 重复利用引理 2(见图 5(a) 与 (b)) 得:

$$
\begin{aligned}
Z(R(G))= & Z\left(R(G)-u e^{*}-v e^{*}-u v\right)+Z\left(R(G)-u-e^{*}\right) \\
& +Z\left(R(G)-v-e^{*}\right)+Z\left(R(G)-u-v-e^{*}\right) .
\end{aligned}
$$

由 $R(G)$ 的定义,

$$
R(G)-u e^{*}-v e^{*}-u v=R(G-u v)=R(G-e) .
$$

注意到 $R(G)-u-e^{*}$ 有 $s$ 个悬挂点 $e_{i_{1}}^{*}, e_{i_{2}}^{*}, \ldots, e_{i_{s}}^{*}, R(G)-v-e^{*}$ 有 $t$ 个悬挂点 $e_{j_{1}}^{*}, e_{j_{2}}^{*}, \ldots, e_{j_{t}}^{*}, R(G)-u-v-e^{*}$ 有 $s+t$ 个悬挂点 $e_{i_{1}}^{*}, \ldots, e_{i_{s}}^{*}, e_{j_{1}}^{*}, \ldots, e_{j_{t}}^{*}$ (见图 $5(\mathrm{~b}))$. 重复利用引理 3 , 可以证明

$$
\begin{aligned}
& Z\left(R\left(G_{u}\right)\right)=2^{s} Z\left(R(G)-u-e^{*}\right) ; \\
& Z\left(R\left(G_{v}\right)\right)=2^{t} Z\left(R(G)-v-e^{*}\right) ;
\end{aligned}
$$




$$
Z\left(R\left(G_{u v}\right)\right)=2^{s+t} Z\left(R(G)-u-v-e^{*}\right) .
$$

因此由 (1) - - (9) 式得

$$
\begin{aligned}
Z(R(G))= & Z\left(R(G)-u e^{*}-v e^{*}-u v\right)+Z\left(R(G)-u-e^{*}\right) \\
& +Z\left(R(G)-v-e^{*}\right)+Z\left(R(G)-u-v-e^{*}\right) \\
= & Z(R(G-e))+2^{-s} Z\left(R\left(G_{u}\right)\right)+2^{-t} Z\left(R\left(G_{v}\right)\right)+2^{-s-t} Z\left(R\left(G_{u v}\right)\right) \\
= & (s+1)(t+1) \prod_{w \in V(G), w \neq u, v}\left(d_{G}(w)+1\right) \\
& +(t+1) \prod_{w \in V(G), w \neq u, v}\left(d_{G}(w)+1\right) \\
& +(s+1) \prod_{w \in V(G), w \neq u, v}\left(d_{G}(w)+1\right)+\prod_{w \in V(G), w \neq u, v}\left(d_{G}(w)+1\right) \\
= & (s+2)(t+2) \prod_{w \in V(G), w \neq u, v}\left(d_{G}(w)+1\right) .
\end{aligned}
$$

注意到 $d_{G}(u)=s+1, d_{G}(v)=t+1$, 因此

$$
(s+2)(t+2) \prod_{w \in V(G), w \neq u, v}\left(d_{G}(w)+1\right)=\left(d_{1}+1\right)\left(d_{2}+1\right) \cdots\left(d_{n}+1\right) .
$$

至此完成了定理的归纳法证明.

为了给出定理 1 的一一对应法证明, 我们需要引入如下一些记号: 设 $G$ $=(V(G), E(G))$ 是一个简单图, 其顶点集为 $V(G)=\left\{v_{1}, v_{2}, \ldots, v_{n}\right\}$, 其边集为 $E(G)=\left\{e_{1}, e_{2}, \ldots, e_{m}\right\} ; R(G)$ 的顶点集为 $V(R(G))=V(G) \cup\left\{e_{i}^{*} \mid 1 \leqslant i \leqslant m\right\}$, 边 集为 $E(R(G))=E(G) \cup\left\{\left(v_{i}, e^{*}\right),\left(v_{j}, e^{*}\right) \mid e=\left(v_{i}, v_{j}\right) \in E(G)\right\}$. 因此, $R(G)$ 含有两 种形式的顶点: $v$ 型顶点与 $e$ 型顶点; $R(G)$ 含有两种形式的边: $(v, v)$ 型边与 $(v, e)$ 型边. 假设 $\mathcal{M}(R(G))$ 表示 $R(G)$ 的所有匹配的集合, 其中空集看成 $R(G)$ 的不含边 的匹配 (零匹配). 如果 $M \in \mathcal{M}(R(G))$, 令 $V\left(M^{c}\right)$ 表示 $R(G)$ 中没有被 $M$ 的边关联 的 $v$ 型顶点的集合, 定义: $M^{*}=M \cup V\left(M^{c}\right), \mathcal{M}^{*}(R(G))=\left\{M^{*} \mid M \in \mathcal{M}(R(G))\right\}$. 由 $\mathcal{M}(R(G))$ 与 $\mathcal{M}^{*}(R(G))$ 的定义, $|\mathcal{M}(R(G))|=\left|\mathcal{M}^{*}(R(G))\right|$, 这是因为 $V\left(M^{c}\right)$ 由 $M$ 唯一地确定. 设 $v_{i}$ 是 $R(G)$ 中的任一个 $v$ 型顶点, 定义 $E_{i}$ 为 $R(G)$ 中与 $v_{i}$ 相关联的所有 $(v, e)$ 型边的集合, 而 $X_{i}=\left\{v_{i}\right\} \cup E_{i}$. 显然 $\left|X_{i}\right|=\left|E_{i}\right|+1=$ $d_{i}+1$, 其中 $d_{i}$ 是 $G$ 中的顶点 $v_{i}$ 的度. 定义 $\mathcal{W}(R(T))=X_{1} \times X_{2} \times \cdots \times X_{n}=$ $\left\{\left(x_{1}, x_{2}, \ldots, x_{n}\right) \mid x_{i} \in X_{i}\right\}$, 因此 $|\mathcal{W}(R(G))|=\left(d_{1}+1\right)\left(d_{2}+1\right) \cdots\left(d_{n}+1\right)$.

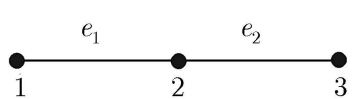

(a)

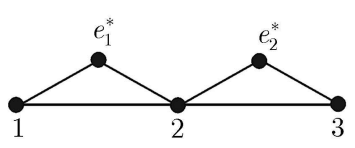

(b)

图 6 (a) 三个顶点的路 $G$, (b) $G$ 相应的图 $R(G)$ 
对于图 6(a) 与 (b) 中的图 $G$ 与 $R(G), E_{1}=\left\{\left(1, e_{1}^{*}\right)\right\}, E_{2}=\left\{\left(2, e_{1}^{*}\right),\left(2, e_{2}^{*}\right)\right\}$, $E_{3}=\left\{\left(3, e_{2}^{*}\right)\right\}, X_{1}=\left\{1,\left(1, e_{1}^{*}\right)\right\}, X_{2}=\left\{2,\left(2, e_{1}^{*}\right),\left(2, e_{2}^{*}\right)\right\}, X_{3}=\left\{3,\left(3, e_{2}^{*}\right)\right\}$, $M=\left\{\left(1, e_{1}^{*}\right),\left(3, e_{2}^{*}\right)\right\}$ 是 $R(G)$ 的一个含有 2 条边的匹配, 由 $M^{*}$ 的定义, $M^{*}=$ $\left\{2,\left(1, e_{1}^{*}\right),\left(3, e_{2}^{*}\right)\right\}$.

定理 1 的一一对应法证明 设 $\mathcal{M}^{*}(R(G))$ 与 $\mathcal{W}(R(G))$ 如上定义，因此， $\left|\mathcal{M}^{*}(R(G))\right|=|\mathcal{M}(R(G))|$. 我们只需要构造一个一一对应 $f: \mathcal{W}(R(G)) \rightarrow$ $\mathcal{M}^{*}(R(G))$. 对任意的 $W=\left(x_{1}, x_{2}, \ldots, x_{n}\right) \in \mathcal{W}(R(G))$, 需要定义 $f(W) \in$ $\mathcal{M}^{*}(R(G))$. 为此考虑下面 3 种情形

(i) 如果 $x_{i}=v_{i}$, 令 $x_{i} \in f(M)$;

(ii) 如果存在 $e_{i j}^{*}$, 使得 $x_{i}=\left(v_{i}, e_{i j}^{*}\right)$, 且 $x_{j}=\left(v_{j}, e_{i j}^{*}\right)$, 令 $\left(v_{i}, v_{j}\right) \in f(W)$;

(iii) 如果存在 $e_{i j}^{*}$, 使得 $x_{i}=\left(v_{i}, e_{i j}^{*}\right)$, 但 $x_{j} \neq\left(v_{j}, e_{i j}^{*}\right)$, 令 $\left(v_{i}, e_{i j}^{*}\right) \in f(W)$, 其 中 $e_{i j}=\left(v_{i}, v_{j}\right)$ 表示 $G$ 中的一条边.

上面 3 种情形完全定义了 $f(W)$, 而且由情形 (ii) 与 (iii) 得到的 $f(W)$ 中的元 素恰好构成 $R(G)$ 中的一个匹配 $M$, 由情形 (i) 得到 $f(W)$ 中的元素恰好是不与 $M$ 关联的 $R(G)$ 中的 $v$ 型顶点集. 不难发现，由 $W$ 构造 $f(W)$ 的过程是可逆的, 因此, $f$ 是 $\mathcal{W}(R(G))$ 与 $\mathcal{M}^{*}(R(G))$ 之间的一个一一对应 (我们将在下面的例子 中说明此一一对应). 至此, 完成了定理的一一对应法证明.

例子 设 $G$ 是 3 个顶点的路， $R(G)$ 是相应的图 (见图 6(a) 与 (b)). 由定义， $\mathcal{M}(R(G))$ 表示 $R(G)$ 的所有匹配的集合, 因此, $\mathcal{M}(R(G))=\left\{M_{i} \mid 0 \leqslant i \leqslant 11\right\}$, 其中 $M_{0}=\Phi, M_{1}=\{(1,2)\}, M_{2}=\{(2,3)\}, M_{3}=\left\{\left(1, e_{1}^{*}\right)\right\}, M_{4}=\left\{\left(2, e_{1}^{*}\right)\right\}, M_{5}=$ $\left\{\left(2, e_{2}^{*}\right)\right\}, M_{6}=\left\{\left(3, e_{2}^{*}\right)\right\}, M_{7}=\left\{\left(1, e_{1}^{*}\right),(2,3)\right\}, M_{8}=\left\{\left(1, e_{1}^{*}\right),\left(3, e_{2}^{*}\right)\right\}, M_{9}=\left\{\left(1, e_{1}^{*}\right)\right.$, $\left.\left(2, e_{2}^{*}\right)\right\}, M_{10}=\left\{(1,2),\left(3, e_{2}^{*}\right)\right\}, M_{11}=\left\{\left(2, e_{1}^{*}\right),\left(3, e_{2}^{*}\right)\right\}$. 由 $M_{i}^{*}$ 的定义 $(0 \leqslant i \leqslant 11)$, $M_{0}^{*}=\{1,2,3\}, M_{1}^{*}=\{3,(1,2)\}, M_{2}^{*}=\{1,(2,3)\}, M_{3}^{*}=\left\{2,3,\left(1, e_{1}^{*}\right)\right\}, M_{4}^{*}=$ $\left\{1,3,\left(2, e_{1}^{*}\right)\right\}, M_{5}^{*}=\left\{1,3,\left(2, e_{2}^{*}\right)\right\}, M_{6}^{*}=\left\{1,2,\left(3, e_{2}^{*}\right)\right\}, M_{7}^{*}=\left\{\left(1, e_{1}^{*}\right),(2,3)\right\}, M_{8}^{*}=$ $\left\{2,\left(1, e_{1}^{*}\right),\left(3, e_{2}^{*}\right)\right\}, M_{9}^{*}=\left\{3,\left(1, e_{1}^{*}\right),\left(2, e_{2}^{*}\right)\right\}, M_{10}^{*}=\left\{(1,2),\left(3, e_{2}^{*}\right)\right\}, M_{11}^{*}=\{1,(2$, $\left.\left.e_{1}^{*}\right),\left(3, e_{2}^{*}\right)\right\} ; E_{1}=\left\{\left(1, e_{1}^{*}\right)\right\}, E_{2}=\left\{\left(2, e_{1}^{*}\right),\left(2, e_{2}^{*}\right)\right\}, E_{3}=\left\{\left(3, e_{2}^{*}\right)\right\} ; X_{1}=\left\{1,\left(1, e_{1}^{*}\right)\right\}$, $X_{2}=\left\{2,\left(2, e_{1}^{*}\right),\left(2, e_{2}^{*}\right)\right\}, X_{3}=\left\{3,\left(3, e_{2}^{*}\right)\right\}$. 由 $W_{i}$ 的定义, $W_{0}=(1,2,3), W_{1}=$ $\left(\left(1, e_{1}^{*}\right),\left(2, e_{1}^{*}\right), 3\right), W_{2}=\left(1,\left(2, e_{2}^{*}\right),\left(3, e_{2}^{*}\right)\right), W_{3}=\left(\left(1, e_{1}^{*}\right), 2,3\right), W_{4}=\left(1,\left(2, e_{1}^{*}\right), 3\right)$, $W_{5}=\left(1,\left(2, e_{2}^{*}\right), 3\right), W_{6}=\left(1,2,\left(3, e_{2}^{*}\right)\right), W_{7}=\left(\left(1, e_{1}^{*}\right),\left(2, e_{2}^{*}\right),\left(3, e_{2}^{*}\right)\right), W_{8}=\left(\left(1, e_{1}^{*}\right)\right.$, $\left.2,\left(3, e_{2}^{*}\right)\right), W_{9}=\left(\left(1, e_{1}^{*}\right),\left(2, e_{2}^{*}\right), 3\right), W_{10}=\left(\left(1, e_{1}^{*}\right),\left(2, e_{1}^{*}\right),\left(3, e_{2}^{*}\right)\right), W_{11}=\left(1,\left(2, e_{1}^{*}\right)\right.$, $\left.\left(3, e_{2}^{*}\right)\right)$. 显然, $\mathcal{W}(R(G))=X_{1} \times X_{2} \times X_{3}=\left\{W_{i} \mid 0 \leqslant i \leqslant 11\right\}, f: W_{i} \longmapsto f\left(W_{i}\right)=$ $M_{i}^{*}$ 是 $\mathcal{W}(R(G))$ 到 $\mathcal{M}^{*}(R(G))$ 的一一对应.

\section{3 结论}

本文证明了图 $G$ 的一个运算图 $R(G)$ 的匹配数完全可以利用 $G$ 的度序列表 示, 一个很自然的问题是: $R(G)$ 的匹配多项式能不能用图 $G$ 的某些参数表示? 
另一个有趣的问题是: 图 $G$ 的全图 $T(G)$ 与线图 $L(G)$ 的匹配数与匹配多项式能 否由图 $G$ 的某些参数表示?

致谢 作者衰心感谢审稿人的意见与建议.

\section{参考文献}

1 Lovász L, Plummer M. Matching Theory. Ann Discrete Math 29. New York: North-Holland, 1986

2 Cvetković D, Doob M, Sachs H. Spectra of Graph-Theory and Applications. New York: Academic Press, 1980

3 Hosoya H. Topological index, a newly proposed quantity characterizing the topological nature of structural isomers of saturated hydrocarbons. Bull Chem Soc Jpn, 1971, 44: 2332-2339

4 Farrell E J. An introduction to matching polynomials. J Combin Theory, Ser B, 1979, 27: 75-86

5 Farrell E J, Wahid S A. D-graphs, I. An introduction to graphs whose matching polynomials are determinants of matrices. Bull Inst Combin Appl, 1995, 15: 81-86

6 Fishermann M, Volkmann L, Rautenbach D. A note on the number of matchings and independent sets in trees. Discrete Appl Math, 2005, 145: 483-489

7 Gruber C, Kunz H. General properties of polymer systems. Comm Math Phys, 1971, 22: 133-161

8 Gutman I, Vidovic D, Furtula B. Coulson function and Hosoya index. Chem Phys Lett, 2002, 355: 378 382

9 Heilmann O J, Lieb E H. Monomers and dimers. Phys Rev Lett, 1970, 24: 1412-1414

10 Randic M. Wiener-Hosoya index-a novel graph theoretical molecular descriptor. J Chem Inf Comput Sci, 2004, 44: 373-377

11 Randic M, Zupan J. On interpretation of well-known topological indices. J Chem Inform Comput Sci, 2001, 41: 550-560

12 Yan W G, Yeh Y N, Zhang F J. On the matching polynomials of graphs with small number of cycles of even length. Intern J Quant Chem, 2005, 105: 124-130

13 Yan W G, Ye L Z. On the minimal energy of trees with a given diameter. Appl Math Lett, 2005, 18: 1046-1025

14 Yan W G, Ye L Z. On the maximal energy and the Hosoya index of a type of trees with many pendant vertices. MATCH Commun Math Comput Chem, 2005, 53: 449-459

15 Zhang L Z, Tian F. Extremal hexagonal chains concerning largest eigenvalue. Sci China Ser A-Math, 2001, 44: 1089-1097

16 Jerrum M. Two-dimensional monomer-dimer systems are computationally intractable. J Stat Phys, 1987, 48: $121-134$

17 Choe Y B, Oxley J G, Sokal A D, et al. Homogeneous multivariate polynomials with the half-plane property. Adv Appl Math, 2004, 32: 88-187

18 Ciucu M. Enumeration of perfect matchings in graphs with reflective symmetry. J Combin Theory, Ser A, 1997, 77: 67-97

19 Hou Y P. On acyclic systems with minimal Hosoya index. Discrete Appl Math, 2003, 119: 251-257

20 Klabjan D, Mohar B. The number of matchings of low order in hexagonal systems. Discrete Math, 1998, 186: $167-175$

21 Lass B. Matching polynomials and duality. Combinatorica, 2004, 24: 427-440

22 Godsil C D. Algebraic Combinatorics. New York: Chapman and Hall, 1993 